\title{
Mitochondrial pyruvate carrier: a potential target for diabetic nephropathy
}

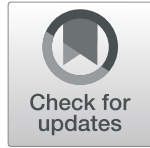

\author{
Huanhuan Zhư ${ }^{\dagger}$ Huiting Wan ${ }^{\dagger}$, Lin Wu', Qing Li, Simeng Liu, Suyan Duan, Zhimin Huang, Chengning Zhang,
}

Bo Zhang, Changying Xing ${ }^{* \dagger}$ and Yanggang Yuan ${ }^{*+}$

\begin{abstract}
Background: Mitochondrial dysfunction contributes to the pathogenesis of diabetic nephropathy (DN). Mitochondrial pyruvate carrier 1 (MPC1) and mitochondrial pyruvate carrier 2 (MPC2) play a bottleneck role in the transport of pyruvate into mitochondrial across the mitochondrial inner membrane. A previous study showed that increasing mitochondrial pyruvate carrier content might ameliorate diabetic kidney disease in $\mathrm{db} / \mathrm{db}$ mice. However, the expression status of MPC1 and MPC2 in patients with DN is unclear.

Methods: Patients with primary glomerulonephropathy (PGN, $n=30)$, PGN with diabetes mellitus (PGN-DM, $n=30$ ) and diabetic nephropathy (DN, $\mathrm{n}=30)$ were included. MPC1 and MPC2 protein levels were examined by immunohistochemistry. The expression of MPC in different groups was evaluated by the Kruskal-Wallis test. Spearman's rank correlation was performed for correlation analysis between MPC levels and clinical factors.

Results: Both MPC1 and MPC2 were localized in renal tubules. Levels of MPC1 and MPC2 were lower in DN patients than in PGN patients and in PGN patients with DM, whereas there were no differences in MPC1 and MPC2 levels among DN stage II to stage IV. Moreover, both MPC1 and MPC2 levels were significantly correlated with serum creatinine, BUN and eGFR in patients with DN, whereas no analogous trend was observed in nondiabetic kidney disease.
\end{abstract}

Conclusions: Our study indicated that MPC localized in renal tubules, which were significantly decreased in DN. MPC was associated with clinical features, especially those representing renal functions.

Keywords: Mitochondrial pyruvate carrier, Mitochondria, Diabetic nephropathy

\section{Background}

Since the enormous increase in the global prevalence of diabetes mellitus (DM) over the past decades, diabetic nephropathy (DN) has transcended glomerulonephritis as the leading cause of the end-stage renal disease in many countries [1-3]. The pathogenesis of $\mathrm{DN}$ is complex and multifactorial. Of note, hyperglycemia is known

\footnotetext{
*Correspondence: cyxing62@126.com; ygyuan@njmu.edu.cn

${ }^{+}$Huanhuan Zhu, Huiting Wan and Lin Wu are co-first authors of this study ${ }^{+}$Changying Xing and Yanggang Yuan are senior authors with the equal contribution in this study

Department of Nephrology, The First Affiliated Hospital of Nanjing Medical University, Jiangsu Province Hospital, 300 Guangzhou Road, Nanjing 210029, Jiangsu Province, P. R. of China
}

to play a vital role in the development and progression of DN. The hyperglycemic state induces an increase in toxic glucose metabolites and mitochondrial dysfunction, and intensive glucose control could slow down the progression of DN $[4,5]$.

The kidney, as a highly metabolic organ, requires amounts of energy to maintain its normal function, which is intimately linked with mitochondria. Congruently, kidneys are rich in mitochondria and proximal tubules contain large numbers of mitochondria for synthesizing protein and reabsorbing metabolites [6, 7]. The reduction of mitochondrial content, increased mitochondrial DNA damage, and disruptions of

(c) The Author(s). 2020 Open Access This article is licensed under a Creative Commons Attribution 4.0 International License, which permits use, sharing, adaptation, distribution and reproduction in any medium or format, as long as you give appropriate credit to the original author(s) and the source, provide a link to the Creative Commons licence, and indicate if changes were made. The images or other third party material in this article are included in the article's Creative Commons licence, unless indicated otherwise in a credit line to the material. If material is not included in the article's Creative Commons licence and your intended use is not permitted by statutory regulation or exceeds the permitted use, you will need to obtain permission directly from the copyright holder. To view a copy of this licence, visit http://creativecommons.org/licenses/by/4.0/ The Creative Commons Public Domain Dedication waiver (http://creativecommons.org/publicdomain/zero/1.0/) applies to the data made available in this article, unless otherwise stated in a credit line to the data. 
mitochondrial networking (including fission and fusion) were observed in DN [6, 8-10]. Moreover, hyperglycemia increased the tricarboxylic acid cycle and altered the glycolytic pathway via the elevated level of advanced glycation end products, the activity of protein kinase $\mathrm{C}$ and hexosamine pathways and so on, which contributed to mitochondrial dysfunction $[8,11]$. There were some investigations of patients and animal models have confirmed that mitochondrial dysfunction was postulated as a primary initiator and played a pivotal role in the progression of DN [12-15]. Hence, therapies that target mitochondrial function would be beneficial to alleviate DN progression, which has been verified by several mitochondria-targeted antioxidants including coenzyme Q10, mitoquinone, MTP-131 and so on [16-18].

Pyruvate, which is located at the branch point between oxidative and anaerobic metabolism, is critical for maintaining the stability of mitochondrial function [19]. Mitochondrial pyruvate carrier (MPC), a specific carrier in the inner mitochondrial membrane, imports pyruvate from the cytoplasm into the mitochondrial matrix. MPC is composed of two interactional subunits MPC1 and MPC2, and the expressions of both MPC proteins are essential for mitochondrial pyruvate transport [20-22]. MPC regulated mitochondrial substrate selection and was essential for several major pathways of carbohydrate, fat, and amino acid metabolism [23]. Depending on different organs, the MPC might play different effects on glucose homeostasis in type 2 diabetes. Hepatocytespecific MPC disruption attenuated hyperglycemia in mice during high fat diet-induced obesity [24]. By contrary, MPC deficiency resulted in elevated blood glucose concentrations, glucose intolerance and reduced glucose-stimulated insulin secretion in pancreatic $\beta$-cells [25]. However, the role of MPC in kidneys of diabetes mellitus patients was still unknown. Therefore, we aimed to explore renal MPC expression in diabetic nephropathy.

\section{Methods}

\section{Patients}

A total of 30 biopsy-proven diabetic nephropathy (DN) patients were enrolled and 30 primary glomerulonephropathy (PGN) patients (membranous nephropathy, MN, $n=7$; IgA nephropathy, IgAN, $n=6$; focal segmental glomerulosclerosis, FSGS, $n=8$; minimal change disease, MCD, $n=9)$ and 30 primary glomerulonephropathy patients with diabetes mellitus (PGN-DM) (MN, $n=11$; IgAN, $n=8$; FSGS, $n=7$; MCD, $n=4$ ) were used as controls. All the patients were admitted to the First Affiliated Hospital of Nanjing Medical University from September 2016 to May 2019. The exclusion criteria were as follows: (1) patients younger than 18 years old; (2) those obtained glomeruli were less than 5; (3) those with acute kidney injury; (4) those with severe organ insufficiency except kidney. The research complied with the Declaration of Helsinki and was approved by the ethical committees of the First Affiliated Hospital of Nanjing Medical University. Informed consent was obtained from each patient.

\section{Measurements}

The following clinical data of patients were collected at the time of renal biopsy: age, gender, duration of diabetes, blood pressure, fasting glucose, glycosylated hemoglobin $\left(\mathrm{HbA}_{1 \mathrm{C}}\right)$, blood urea nitrogen $(\mathrm{BUN})$, serum creatinine, neutrophil gelatinase-associated lipocalin (NGAL), urine albumin, urine albumin-to-creatinine ratio (ACR) and so on. The urinary acidification function was measured using a ZDJ-4B automatic potentiometric titrator, which described in our previous study [26]. Estimated glomerular filtration rate (eGFR) was calculated according to the Chronic Kidney Disease Epidemiology Collaboration (CKD-EPI) formula [27].

Renal specimens were routinely examined by two experienced pathologists through light microscopy, immunofluorescence and electron microscopy. Diagnosis and classification of DN were made according to the criteria of Tervaert et al [28]. The glomerular classification was as follows: class I, glomerular basement membrane thickening and no specific; class IIa, mild mesangial expansion; class IIb, severe mesangial expansion; class III, nodular sclerosis; and class IV, global glomerulosclerosis in more than $50 \%$ of glomeruli. Diagnosis of MN, IgAN, FSGS, and MCD was made according to international standards.

\section{Immunohistochemistry}

Paraffin sections were deparaffinized and rehydrated. Then, the sections were performed in citrate buffer $(\mathrm{pH}$ 6.0) for $10 \mathrm{~min}$ and washed with phosphate-buffered saline. After blocking by $5 \%$ bovine serum albumin for $1 \mathrm{~h}$ at room temperature, the sections were incubated with MPC1 antibody (1:900, Sigma, HPA045119) and MPC2 antibody (1: 20, Sigma, HPA056091) overnight at $4{ }^{\circ} \mathrm{C}$. The sections were washed with PBS and then incubated with the second antibody for $1 \mathrm{~h}$ at $37^{\circ} \mathrm{C}$. The sections were then stained with $3,3^{\prime}$-diaminobenzidine tetrahydrochloride for $10 \mathrm{~min}$ and counterstained with hematoxylin, dehydrated and mounted. Finally, each section was randomly captured $8-10$ photographs by the microscope and calculated by the Image-Pro Plus.

\section{Statistical analysis}

SPSS 22.0 software (SPSS, Chicago, IL, USA) was used for data analysis. Variables were expressed as the mean \pm standard deviation. Comparisons for normal distribution groups were performed using the one-way analysis of 
variance. Differences among non-normal distribution groups were analyzed using the Kruskal-Wallis test. Correlations between MPC and clinical characteristics were assessed by using Spearman's rank test. Significance was defined as $p<0.05$.

\section{Results}

\section{Patients characteristics}

A total of 90 patients were included in this study and categorized into three groups according to the renal biopsy: those with biopsy-proven diabetic nephropathy (DN group, $n=30$ ), those with biopsy-proven primary glomerulonephropathy (PGN group, $n=30$ ), and those with biopsy-proven PGN and with diabetes mellitus (PGN-DM group, $n=30$ ). The baseline characteristics of patients were shown in Table 1 . Gender and age were comparable among groups. Of the DN patients, 6 (20\%) were female, and their mean age was $50.57 \pm 11.71$ years, and the mean duration of diabetes was $9.94 \pm 6.77$ years. The estimated GFR was $59.64 \pm 33.42 \mathrm{ml} / \mathrm{min} / 1.73 \mathrm{~m}^{2}$ at baseline and proteinuria was $5.50 \pm 5.10 \mathrm{~g} / 24 \mathrm{~h}$.

Diabetic nephropathy patients had longer diabetes duration $(p<0.001)$, higher levels of fasting glucose $(p<$ $0.001)$ and $\mathrm{HbA}_{1 \mathrm{C}}(p=0.002)$. The serum creatinine $(p<$ $0.001)$, BUN $(p<0.001)$ and cystatin $C(p=0.002)$ levels were significantly higher in the DN group, whereas eGFR $(p<0.001)$ was lower. And there were also significant differences in urinary albumin $(p=0.037)$ and ACR $(p<$ $0.001)$ among groups. Moreover, lower urinary titratable acid $(p=0.011)$ and ammonia $(p<0.001)$ were observed in the DN group. Therefore, serum fasting glucose, $\mathrm{HbA}_{1 \mathrm{C}}$, creatinine, BUN, cystatin C, eGFR, urinary albumin, ACR, urinary titratable acid, and urinary ammonia were parameters that were chosen for further analysis.

Table 1 Baseline characteristics in patients $(n=90)$

\begin{tabular}{|c|c|c|c|c|}
\hline Characteristics & $\begin{array}{l}\text { PGN } \\
(n=30)\end{array}$ & $\begin{array}{l}\text { PGN with DM } \\
(n=30)\end{array}$ & $\begin{array}{l}\mathrm{DN} \\
(\mathrm{n}=30)\end{array}$ & $p$ value \\
\hline Female, n (\%) & $11(36.7 \%)$ & $10(33.3 \%)$ & $6(20 \%)$ & 0.274 \\
\hline Age (years) & $47.17 \pm 13.60$ & $51.73 \pm 14.06$ & $50.57 \pm 11.71$ & 0.382 \\
\hline Diabetes duration (years) & 0 & $3.77 \pm 3.87$ & $9.94 \pm 6.77$ & $<0.001$ \\
\hline Hypertension (years) & $3.07 \pm 3.94$ & $3.77 \pm 5.73$ & $6.19 \pm 7.45$ & 0.105 \\
\hline Hypertension, n (\%) & 15 (50\%) & $16(53.3 \%)$ & $24(80 \%)$ & 0.033 \\
\hline SBP $(\mathrm{mmHg})$ & $133.73 \pm 16.26$ & $134.00 \pm 17.66$ & $146.00 \pm 25.19$ & 0.030 \\
\hline $\mathrm{DBP}(\mathrm{mmHg})$ & $85.07 \pm 11.72$ & $84.00 \pm 9.69$ & $88.47 \pm 13.48$ & 0.310 \\
\hline $\mathrm{Hb}(\mathrm{g} / \mathrm{L})$ & $139.90 \pm 14.11$ & $137.40 \pm 17.10$ & $109.07 \pm 22.51$ & $<0.001$ \\
\hline $\mathrm{HbA}_{1 \mathrm{C}}(\%)$ & $5.61 \pm 0.59$ & $7.15 \pm 1.30$ & $7.57 \pm 1.94$ & 0.002 \\
\hline Fasting glucose (mmol/L) & $4.94 \pm 0.96$ & $6.57 \pm 1.84$ & $8.20 \pm 4.95$ & $<0.001$ \\
\hline Total cholesterol (mg/dL) & $6.47 \pm 3.53$ & $6.85 \pm 2.78$ & $5.66 \pm 1.74$ & 0.293 \\
\hline LDL cholesterol (mg/dL) & $4.20 \pm 2.39$ & $4.46 \pm 1.90$ & $3.80 \pm 1.30$ & 0.180 \\
\hline HDL cholesterol (mg/dL) & $1.34 \pm 0.45$ & $1.27 \pm 0.41$ & $1.16 \pm 0.39$ & 0.396 \\
\hline Triglyceride (mg/dL) & $2.10 \pm 1.51$ & $3.40 \pm 2.40$ & $1.96 \pm 1.84$ & 0.004 \\
\hline Albumin (g/L) & $32.93 \pm 11.12$ & $29.79 \pm 9.67$ & $32.24 \pm 6.19$ & 0.388 \\
\hline Serum creatinine (umol/L) & $73.24 \pm 21.51$ & $84.65 \pm 51.27$ & $155.84 \pm 95.87$ & $<0.001$ \\
\hline $\operatorname{eGFR}\left(\mathrm{ml} / \mathrm{min} / 1.73 \mathrm{~m}^{2}\right)$ & $100.10 \pm 20.16$ & $92.15 \pm 27.99$ & $59.64 \pm 33.42$ & $<0.001$ \\
\hline Serum cystatin C (mg/L) & $1.07 \pm 0.27$ & $1.28 \pm 0.51$ & $2.05 \pm 0.96$ & 0.002 \\
\hline $\mathrm{BUN}(\mathrm{mmol} / \mathrm{L})$ & $5.44 \pm 1.18$ & $5.79 \pm 2.06$ & $10.63 \pm 6.19$ & $<0.001$ \\
\hline Uric acid (umol/L) & $370.60 \pm 87.13$ & $387.97 \pm 83.67$ & $405.54 \pm 103.96$ & 0.344 \\
\hline Serum NGAL (ng/mL) & $169.18 \pm 96.86$ & $238.54 \pm 112.51$ & $227.65 \pm 149.28$ & 0.053 \\
\hline Urine NGAL (ng/mL) & $60.99 \pm 116.73$ & $95.43 \pm 186.92$ & $96.73 \pm 146.77$ & 0.394 \\
\hline Urine albumin(g/24 h) & $3.44 \pm 4.59$ & $4.97 \pm 4.62$ & $5.50 \pm 5.10$ & 0.037 \\
\hline Urine ACR (mg/g) & $119.71 \pm 110.37$ & $287.55 \pm 146.89$ & $465.32 \pm 304.63$ & $<0.001$ \\
\hline Urine $\mathrm{pH}$ & $6.09 \pm 0.57$ & $5.89 \pm 0.56$ & $5.94 \pm 0.66$ & 0.398 \\
\hline Urine bicarbonate $(\mathrm{mmol} / \mathrm{L})$ & $24.70 \pm 2.42$ & $24.63 \pm 1.57$ & $25.26 \pm 2.21$ & 0.324 \\
\hline Urine titratable acid $(\mathrm{mmol} / \mathrm{L})$ & $24.03 \pm 16.62$ & $18.26 \pm 10.12$ & $12.59 \pm 9.78$ & 0.011 \\
\hline Urine ammonia (mmol/L) & $44.67 \pm 26.83$ & $31.74 \pm 18.89$ & $18.48 \pm 12.83$ & $<0.001$ \\
\hline
\end{tabular}




\section{MPC expression in the kidney}

Both MPC1 and MPC2 expression were confined to the cytoplasm of tubular cells. Typical cytoplasmic staining of MPC1 and MPC2 were shown in Fig. 1 and Fig. 2. Analysis of immunohistochemical staining showed that MPC1 expression was significantly lower in the DN group than the PGN-DM and the PGN group (both $p<$ 0.001), and the PGN-DM group also had lower expression of MPC1 than the PGN group $(p<0.001)$ (Fig. 3a). The expression of MPC2 was consistent with MPC1 (Fig. 3e). No significant difference was observed in the expression of MPC1 and MPC2 among different pathological stages of $\mathrm{DN}(p=0.099, p=0.385$, respectively) (Fig. 3d and h). Furthermore, we found no statistical differences of MPC1 and MPC2 expression in different types of pathology in the PGN-DM group ( $p=0.9, p=$ 0.915 , respectively) (Fig. $3 \mathrm{c}$ and g), and a similar trend was also noted in the PGN group $(p=0.757, p=0.573$, respectively) (Fig. 3b and f).

\section{Association between MPC and clinical parameters}

As shown in Table 2 and Fig. 4, in patients with diabetic nephropathy, the MPC1 was inversely correlated with serum creatinine $(r=-0.411, p=$ $0.024)$ and BUN $(r=-0.619, p<0.001)$, whereas it was positively correlated with hemoglobin $(r=0.402$, $p=0.028)$ and eGFR $(r=0.474, p=0.008)$. And the MPC2 was negatively correlated with serum creatinine $(r=-0.387, p=0.034)$ and BUN $(r=-0.578, p=$ $0.001)$, while it was positively correlated with $\mathrm{HbA1c}$ $(r=0.437, p=0.029)$ and eGFR $(r=0.383, p=0.037)$. There was no association between MPC and other clinical characteristics, including serum fasting glucose, cystatin $\mathrm{C}$, urinary albumin, ACR, urinary titratable acid, and urinary ammonia, which showed significant differences among $\mathrm{DN}$ and control groups. Moreover, we assessed correlations of MPC expression and clinical features in the PGN-DM group. The negative correlation was found between MPC1 and urinary albumin $(r=-0.447, p=0.013)$. And there were positive correlations between MPC2 and age $(r=0.485, p=0.007)$, and HDL $(r=0.374$, $p=0.042)$. There were no significant correlations between MPC and serum creatinine, BUN, and eGFR in the PGN-DM group, which showed significant correlations in the DN group.

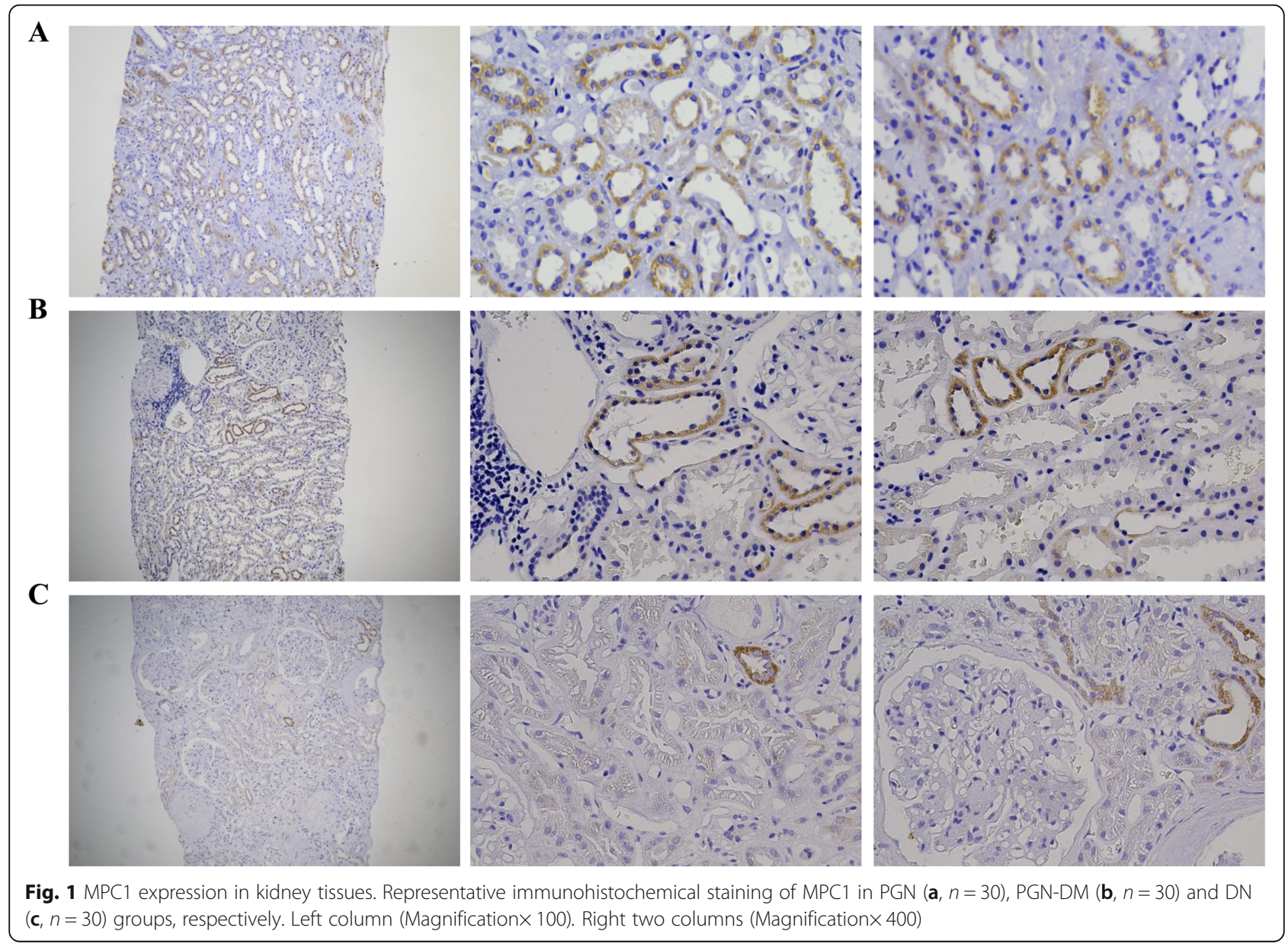




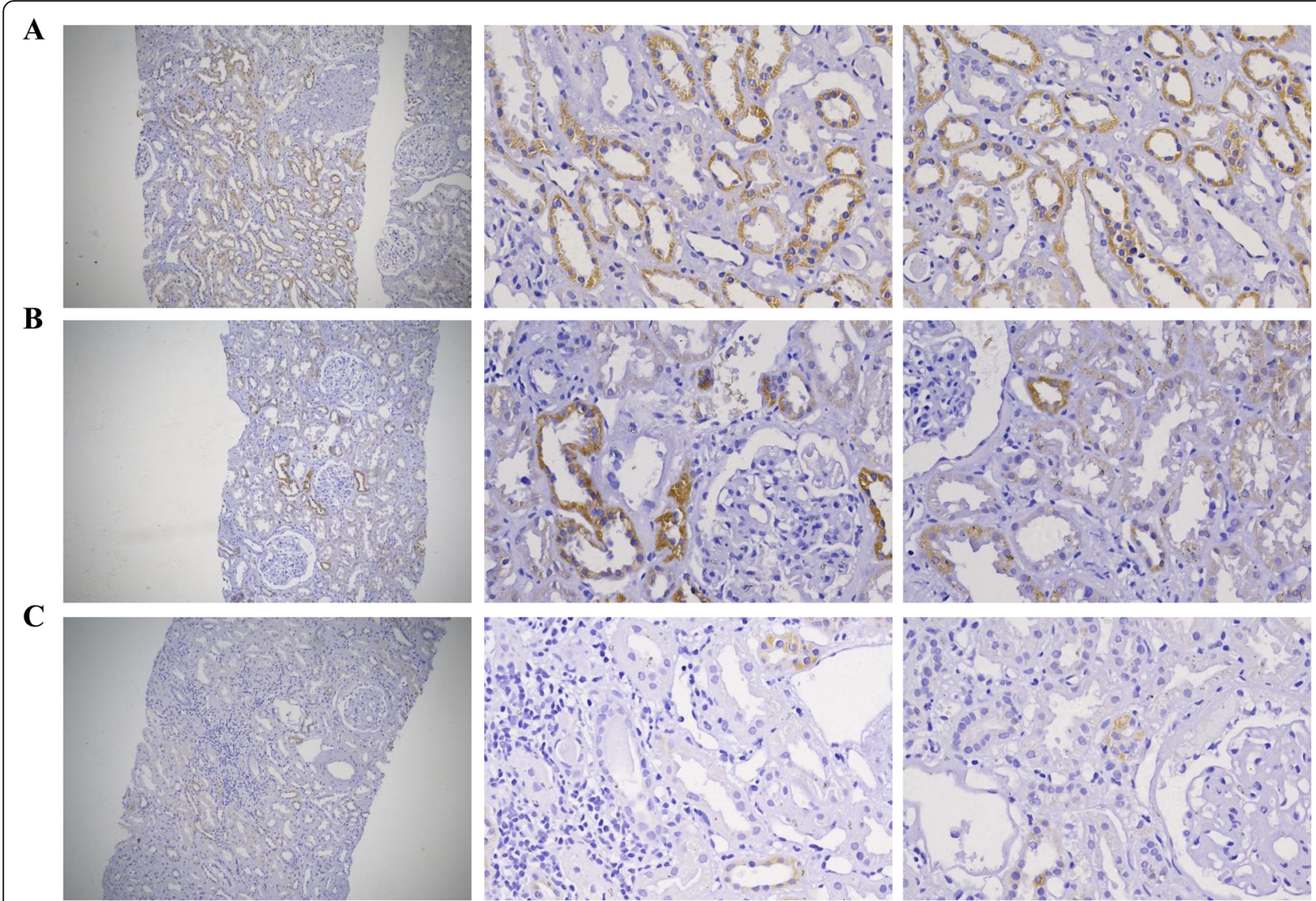

Fig. 2 MPC2 expression in kidney tissues. Representative immunohistochemical staining of MPC2 in PGN (a, $n=30), P G N-D M(\mathbf{b}, n=30)$ and DN $(\mathbf{c}, n=30)$ groups, respectively. Left column (Magnification $\times 100)$. Right two columns (Magnification $\times 400$ )

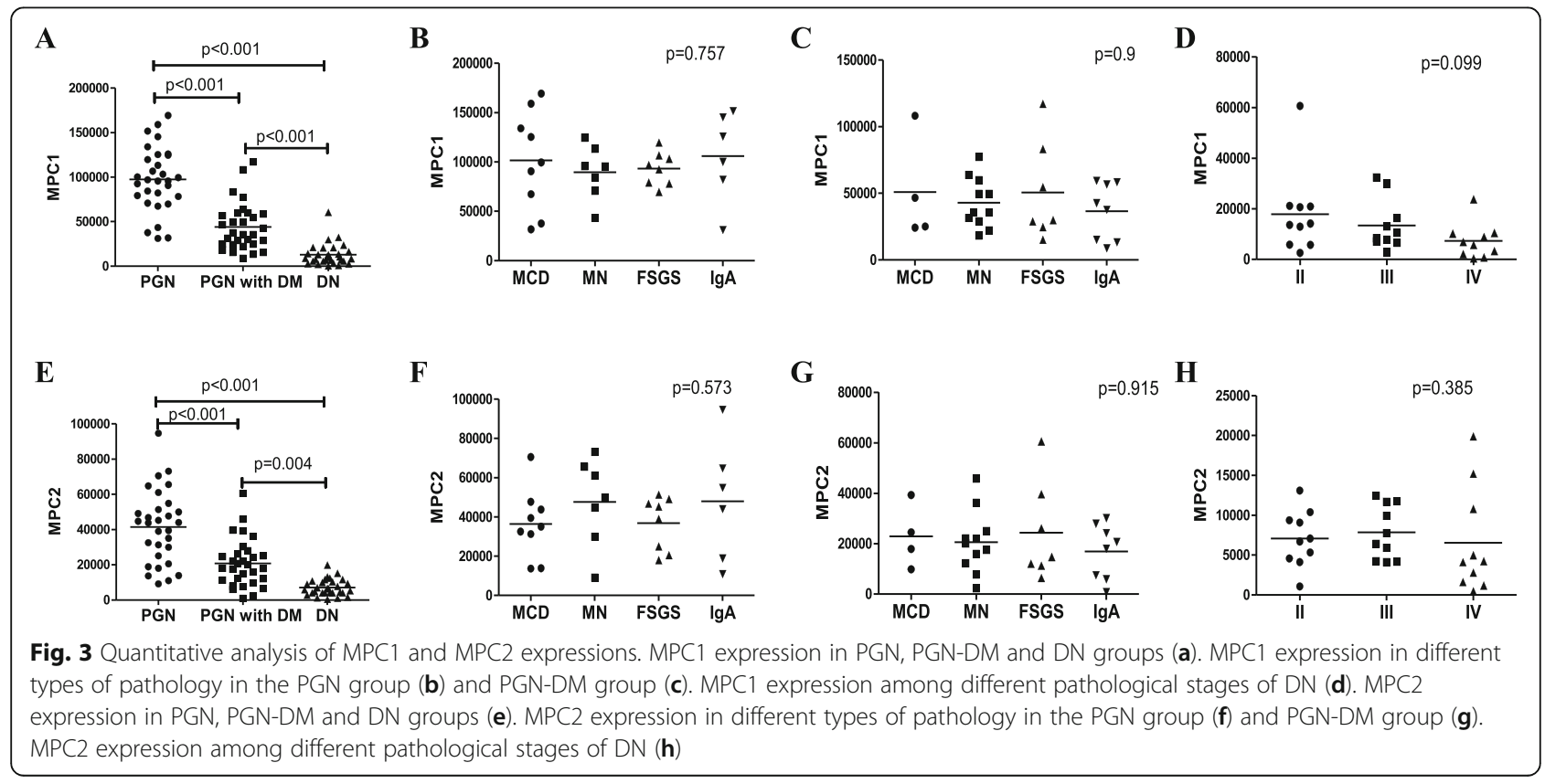


Table 2 Associations between MPC and clinical features in DN and PGN-DM

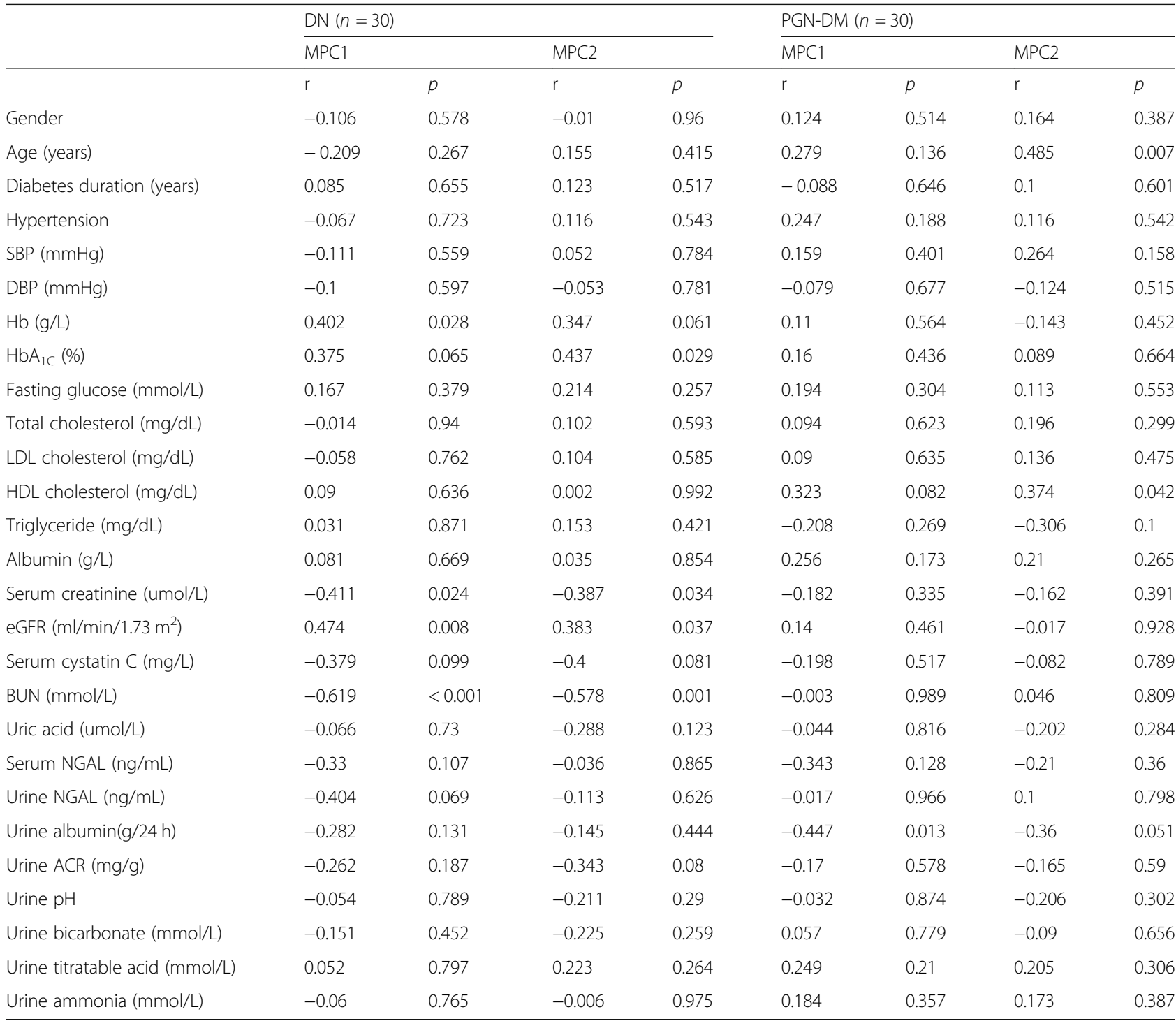

\section{Discussion}

Mitochondrial dysfunction contributes to the development and progression of diabetic nephropathy, which is also related to renal injury in non-diabetic kidney diseases. However, the pathogenic mechanisms of mitochondrial dysfunction are still not incompletely understood in DN. In this study, we focused on associations between mitochondria-related MPC and kidney in diabetes and diabetic nephropathy. We found that both MPC1 and MPC2 expression in kidneys were significantly lower in the DN group than the PGN-DM and the PGN group, whereas there were no differences in MPC levels among DN stage II to stage IV. Moreover, both MPC1 and MPC2 levels were significantly correlated with serum creatinine, BUN and eGFR in patients with $\mathrm{DN}$, whereas no analogous trend was observed in the non-diabetic kidney disease group.
The MPC is a gatekeeper in mitochondrial metabolism for transporting pyruvate, which is a central substrate in carbohydrate, fatty acid, and amino acid metabolism [19-22]. The MPC has been implicated in many diseases and exerted different expressions and effects in various disease models. Several studies have indicated that multiple cancers exhibited diminished MPC expression and activity, which causing dysfunction of mitochondrial pyruvate uptake. High glycolysis of tumor cells is known as the Warburg effect and the MPC is likely involved in the Warburg effect, which offers a potential target for cancer therapy [29-31]. Growing evidence supported that the altered mitochondrial function and the Warburg effect were closely correlated in the development of DN [32]. In our study, we found that MPC expression decreased in nondiabetic kidney disease coincident with diabetes mellitus and diabetic nephropathy, while 


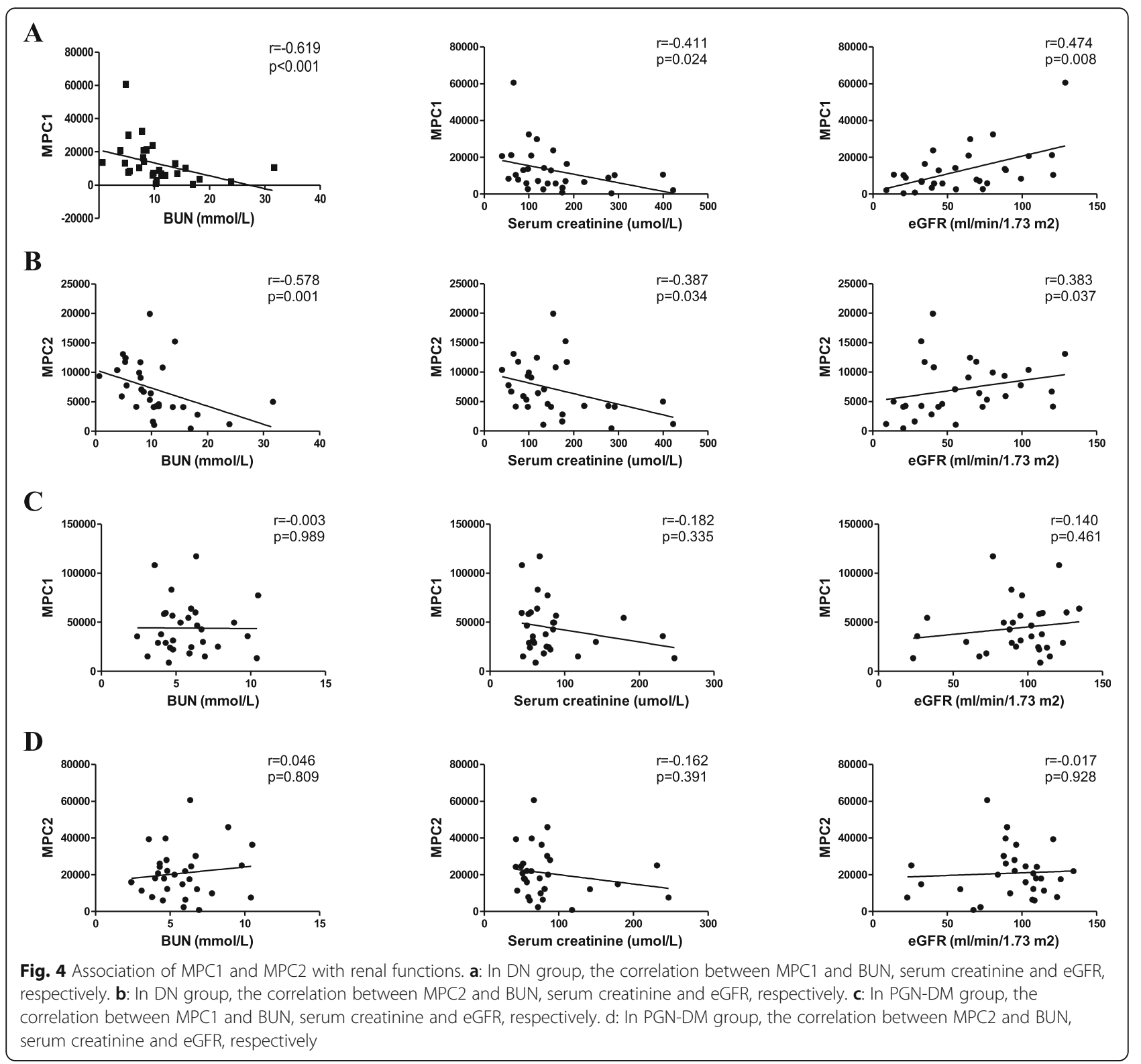

diminished MPC expression was more serious in DN than nondiabetic kidney disease with DM. These findings were consistent with a recent study that the loss of renal MPC content was observed in $\mathrm{db} / \mathrm{db}$ mice [33]. The mechanisms regulating MPC are incompletely understood. Thiazolidinediones, a widely used class of insulin sensitizers, were shown to bind to and inhibit the MPC [25]. The findings in liver, muscle and neurons have demonstrated that inhibition of MPC activities could be contextually beneficial in diseased conditions [34]. However, Feng et al. demonstrated that there was no different MPC expression in high glucose-treated podocytes compared to that in the control group, inhibition of MPC2 in podocytes led to pyruvate accumulation, mitochondrial dysfunction and cell apoptosis [35].
Regardless of loss in the expression or the activity of MPC, it all came with the reduced pyruvate transport, but whether this change was beneficial or pernicious might depend on types of disease. SIRT3, which is an $\mathrm{NAD}^{+}$-dependent deacetylase in mitochondria, targets several diverse enzymes involved in the regulation of the antioxidative defense system, metabolism and longevity [36]. SIRT3 activity corelated with the maintenance of mitochondrial energy homeostasis and antioxidant defense in proximal and distal tubule compartments [37]. The kidney of diabetic mice displayed time dependent reduction in the SIRT3 protein [38]. Also, high glucose decreased the protein and mRNA expression of SIRT3 in a time-dependent manner in cultured tubular epithelial cells [39]. A recent study showed that 
deacetylation modification by SIRT3 could influence MPC1 expression and activity likely via the inhibition of MPC1 degradation [40]. It is possible that the decrease of MPC might also be induced by SIRT3 reduction in diabetic renal tubules, which would partially explain why MPC was specifically reduced in DN with no difference in other glomerular kidney diseases. This study is an initial period for exploring changes of MPC in diabetic nephropathy, and further animal and cell researches are required.

Tubular damage occurs early in DN and may play a critical role in the progression of kidney disease [41]. Of note, renal tubules have a high density of mitochondria and increased glycolysis and glucose oxidation occur in tubules under hyperglycaemic conditions. In the setting of high glucose, tubular injury caused by mitochondrial dysfunction has been highlighted in the pathogenesis and development of DN [6]. Consistently, our present study showed that both MPC1 and MPC2 expressions were significantly lower in DN and correlated with serum creatinine, BUN and eGFR in patients with DN. Less clear is how decreased tubular MPC correlated with the glomerular injury. One explanation for this was reported by a previous study showing that proximal tubules could retrogradely interact with glomeruli and induce glomerular injury. However, there was no obvious association between renal MPC content and tubular marks as urinary NGAL, titratable acid, and ammonia in the present study. Although KIM-1 and NGAL are wellknown tubular injury markers and previous study examined the expressions of tubular markers in DN and confirmed the associations between tubular expressions of NGAL and KIM-1 and renal function decline in DN, serum or urine NGAL levels as biomarkers is still limited since the NGAL protein, which would be affected by inflammation, is not specific to kidney injury [42]. Moreover, Mori $\mathrm{K}$ et al. presented a review that NGAL was a result of the active damage since atrophic nephrons did not produce NGAL [43]. Previous study reported that a sodium-glucose cotransporter-2 (SGLT-2) inhibitor, dapagliflozin, decreased urinary KIM-1 excretion, but did not affect urinary NGAL and LFAPB excretion. SGLT-2 expression was increased in diabetic patients and SGLT-2 inhibition reduced sodium and glucose reabsorption in the proximal tubule, thereby decreasing proximal tubular metabolic workload [44]. Further studies are warranted to determine whether the expression of MPC is correlated with other tubular injury markers.

MPC is fundamentally important in keeping glucose homeostasis. The roles of MPC in regulating glucose homeostasis are multivariate. MPC inhibition in the pancreas contributed to glucose intolerant, while inhibition of the MPC in muscle or liver might ameliorate glucose tolerance [45]. The kidney is an important player in regulating glucose homeostasis through its utilization, gluconeogenesis, and reabsorption in the proximal renal tubule via sodium glucose cotransporters [46]. However, no notable association between MPC expression and glucose was observed in this study. The roles of decreased MPC expression in DN for glucose homeostasis are poorly understood.

This was the first study validating the loss of MPC in the renal tissue of $\mathrm{DM}$ and $\mathrm{DN}$, and illustrating obvious correlations between MPC and renal function in DN. The major limitation of the present study was that the lack of insufficient indicators to test pyruvate and mitochondrial function and changes in the MPC-pyruvatemitochondria axis remained unclear. Moreover, the causal relationship and mechanism between MPC disruption and DN were not explored. Further studies will be required to elucidate the precise mechanisms.

\section{Conclusion}

In summary, decreased MPC content was more pronounced in diabetic nephropathy than nondiabetic kidney disease. Furthermore, MPC levels were significantly correlated with renal functions in patients with DN. Further studies are warranted to determine whether MPC would be a therapeutic target in $\mathrm{DN}$.

\section{Abbreviations \\ DN: Diabetic nephropathy; MPC1: Mitochondrial pyruvate carrier 1; MPC2: Mitochondrial pyruvate carrier 2; PGN: Primary glomerulonephropathy; DM: Diabetes mellitus; MN: Membranous nephropathy; IgAN: IgA nephropathy; FSGS: focal segmental glomerulosclerosis; MCD: Minimal change disease; SBP: Systolic blood pressure; DBP: Diastolic blood pressure; $\mathrm{HbA}_{1}$ : Glycosylated hemoglobin; BUN: Blood urea nitrogen; LDL: Low density lipoprotein; HDL: High density lipoprotein; NGAL: Neutrophil gelatinase-associated lipocalin; ACR: Albumin-to-creatinine ratio; eGFR: Estimated glomerular filtration rate; CKD-EPI: Chronic kidney disease epidemiology collaboration; SGLT-2: Sodium-glucose cotransporter-2}

\section{Acknowledgments}

The authors thank the patients for participation in our study and the staff of the Department of Nephrology at the First Affiliated Hospital of Nanjing Medical University for assistance.

\section{Authors' contributions}

$Y Y$ and $C X$ designed the research. $H Z, L W, H W, Z H$, and BZ conducted the research. $H Z, Q L$, and $S L$ analyzed the data and performed statistical analysis. $H Z, S D, C Z$, and $Y Y$ wrote the manuscript and had responsibility for its final content. $Y Y$ is the guarantor of this work, has complete access to all the data in the study, and takes ultimate responsibility for the study design and integrity of data analysis. All authors have read the final manuscript and approved the submission.

\section{Funding}

This work was financial supported by grants from the National Natural Science Foundation of China (No. 81870469, 81670628, 81300573), the Natural Science Foundation of Jiangsu Province (No. BK20131030), the China Scholarship Council (CSC, File No. 201608320124), Chinese Society of Nephrology (17010060675), the Clinic Research Center of Jiangsu Province (No. BL2014080) and the Priority Academic Program Development (PAPD) of Jiangsu Higher Education Institution. These funding sources were not involved in the design of the study and data collection, analysis, or interpretation. They also played no role in the writing of the manuscript. 


\section{Availability of data and materials}

The datasets analyzed during the current study are available from the corresponding author on reasonable request.

\section{Ethics approval and consent to participate}

All procedures performed in studies involving human participants were in accordance with the ethical standards of the ethics committee of the First Affiliated Hospital of Nanjing Medical University and with the 1964 Helsinki declaration and its later amendments or comparable ethical standards. Informed consent was obtained from all individual participants included in the study.

\section{Consent for publication}

Not applicable.

\section{Competing interests}

The authors declare that they have no competing interests.

\section{Received: 10 March 2020 Accepted: 6 July 2020}

Published online: 14 July 2020

\section{References}

1. Fu H, Liu S, Bastacky SI, Wang X, Tian X-J, Zhou D. Diabetic kidney diseases revisited: a new perspective for a new era. Mol Metab. 2019;30:250-63.

2. Anders H-J, Huber TB, Isermann B, Schiffer M. CKD in diabetes: diabetic kidney disease versus nondiabetic kidney disease. Nat Rev Nephrol. 2018; 14(6):361-77.

3. Alicic RZ, Rooney MT, Tuttle KR. Diabetic kidney disease: challenges, Progress, and possibilities. Clin J Am Soc Nephrol. 2017;12(12):2032-45.

4. Qi W, Keenan HA, Li Q, Ishikado A, Kannt A, Sadowski T, Yorek MA, Wu H, Lockhart S, Coppey LJ, et al. Pyruvate kinase M2 activation may protect against the progression of diabetic glomerular pathology and mitochondrial dysfunction. Nat Med. 2017;23(6):753-62.

5. Thomas MC, Brownlee M, Susztak K, Sharma K, Jandeleit-Dahm KAM, Zoungas S, Rossing P, Groop P-H, Cooper ME. Diabetic kidney disease. Nat Rev Dis Primers. 2015:1:15018.

6. Forbes JM, Thorburn DR. Mitochondrial dysfunction in diabetic kidney disease. Nat Rev Nephrol. 2018;14(5):291-312.

7. Forbes JM. Mitochondria-power players in kidney function? Trends Endocrinol Metab. 2016:27(7):441-2.

8. Bhargava P, Schnellmann RG. Mitochondrial energetics in the kidney. Nat Rev Nephrol. 2017;13(10):629-46.

9. Qi H, Casalena G, Shi S, Yu L, Ebefors K, Sun Y, Zhang W, D'Agati V, Schlondorff D, Haraldsson B, et al. Glomerular endothelial mitochondrial dysfunction is essential and characteristic of diabetic kidney disease susceptibility. Diabetes. 2017;66(3):763-78.

10. Sharma K, Karl B, Mathew AV, Gangoiti JA, Wassel CL, Saito R, Pu M, Sharma S, You Y-H, Wang L, et al. Metabolomics reveals signature of mitochondrial dysfunction in diabetic kidney disease. J Am Soc Nephrol. 2013;24(11):190112.

11. Hallan S, Sharma K. The role of mitochondria in diabetic kidney disease. Curr Diab Rep. 2016;16(7):61.

12. Jiang $H$, Shao $X$, Jia $S$, Qu L, Weng $C$, Shen $X$, Wang $Y$, Huang $H$, Wang $Y$, Wang $C$, et al. The mitochondria-targeted metabolic tubular injury in diabetic kidney disease. Cell Physiol Biochem. 2019;52(2):156-71.

13. Coughlan MT, Nguyen T-V, Penfold SA, Higgins GC, Thallas-Bonke V, Tan SM, Van Bergen NJ, Sourris KC, Harcourt BE, Thorburn DR, et al. Mapping timecourse mitochondrial adaptations in the kidney in experimental diabetes. Clin Sci. 2016;130(9):711-20.

14. Czajka A, Ajaz S, Gnudi L, Parsade CK, Jones P, Reid F, Malik AN. Altered mitochondrial function, mitochondrial DNA and reduced metabolic flexibility in patients with diabetic nephropathy. EBioMedicine. 2015;2(6): 499-512.

15. Zhan M, Usman IM, Sun L, Kanwar YS. Disruption of renal tubular mitochondrial quality control by Myo-inositol oxygenase in diabetic kidney disease. J Am Soc Nephrol. 2015;26(6):1304-21.

16. Chacko BK, Reily C, Srivastava A, Johnson MS, Ye Y, Ulasova E, Agarwal A, Zinn KR, Murphy MP, Kalyanaraman B, et al. Prevention of diabetic nephropathy in Ins2(+/)-(AkitaJ) mice by the mitochondria-targeted therapy MitoQ. Biochem J. 2010;432(1):9-19.
17. Alam NM, Mills WC, Wong AA, Douglas RM, Szeto HH, Prusky GT. A mitochondrial therapeutic reverses visual decline in mouse models of diabetes. Dis Model Mech. 2015;8(7):701-10.

18. Sourris KC, Harcourt BE, Tang PH, Morley AL, Huynh K, Penfold SA, Coughlan MT, Cooper ME, Nguyen TV, Ritchie RH, et al. Ubiquinone (coenzyme Q10) prevents renal mitochondrial dysfunction in an experimental model of type 2 diabetes. Free Radic Biol Med. 2012;52(3):716-23.

19. Gray LR, Tompkins SC, Taylor EB. Regulation of pyruvate metabolism and human disease. Cell Mol Life Sci. 2014;71(14):2577-604.

20. Tavoulari S, Thangaratnarajah C, Mavridou V, Harbour ME, Martinou J-C, Kunji ER. The yeast mitochondrial pyruvate carrier is a hetero-dimer in its functional state. EMBO J. 2019;38(10):e100785.

21. Bricker DK, Taylor EB, Schell JC, Orsak T, Boutron A, Chen Y-C, Cox JE, Cardon CM, Van Vranken JG, Dephoure N, et al. A mitochondrial pyruvate carrier required for pyruvate uptake in yeast, Drosophila, and humans. Science. 2012;337(6090):96-100.

22. Herzig S, Raemy E, Montessuit S, Veuthey J-L, Zamboni N, Westermann B, Kunji ERS, Martinou J-C. Identification and functional expression of the mitochondrial pyruvate carrier. Science. 2012;337(6090):93-6.

23. Halestrap AP. The mitochondrial pyruvate carrier: has it been unearthed at last? Cell Metab. 2012;16(2):141-3.

24. Rauckhorst AJ, Gray LR, Sheldon RD, Fu X, Pewa AD, Feddersen CR, Dupuy AJ, Gibson-Corley KN, Cox JE, Burgess SC, et al. The mitochondrial pyruvate carrier mediates high fat diet-induced increases in hepatic TCA cycle capacity. Mol Metab. 2017;6(11):1468-79.

25. McCommis KS, Hodges WT, Bricker DK, Wisidagama DR, Compan V, Remedi MS, Thummel CS, Finck BN. An ancestral role for the mitochondrial pyruvate carrier in glucose-stimulated insulin secretion. Mol Metabol. 2016;5(8):602-14.

26. Zhu H, Liu X, Zhang C, Li Q, An X, Liu S, Wu L, Zhang B, Yuan Y, Xing C. Association of urinary acidification function with the progression of diabetic kidney disease in patients with type 2 diabetes. J Diabetes Complicat. 2019; 33(11):107419.

27. Levey AS, Stevens LA, Schmid CH, Zhang YL, Castro AF, Feldman HI, Kusek JW, Eggers $P$, Van Lente F, Greene T, et al. A new equation to estimate glomerular filtration rate. Ann Intern Med. 2009;150(9):604-12.

28. Tervaert TWC, Mooyaart AL, Amann K, Cohen AH, Cook HT, Drachenberg $C B$, Ferrario F, Fogo AB, Haas M, de Heer E, et al. Pathologic classification of diabetic nephropathy. J Am Soc Nephrol. 2010;21(4):556-63.

29. Rauckhorst AJ, Taylor EB. Mitochondrial pyruvate carrier function and cancer metabolism. Curr Opin Genet Dev. 2016;38:102-9.

30. Bensard CL, Wisidagama DR, Olson KA, Berg JA, Krah NM, Schell JC, Nowinski SM, Fogarty S, Bott AJ, Wei P, et al. Regulation of Tumor Initiation by the Mitochondrial Pyruvate Carrier. Cell Metab. 2020;31(2):284-300.

31. Park S, Safi R, Liu X, Baldi R, Liu W, Liu J, Locasale JW, Chang C-Y, DP MD. Inhibition of ERRa Prevents mitochondrial pyruvate uptake exposing NADPH-Generating pathways as targetable vulnerabilities in breast cancer. Cell Rep. 2019:27(12):3587-601.

32. Zhang G, Darshi M, Sharma K. The Warburg effect in diabetic kidney disease. Semin Nephrol. 2018;38(2):111-20.

33. Han P, Wang Y, Zhan H, Weng W, Yu X, Ge N, Wang W, Song G, Yi T, Li S, Shao $\mathrm{M}$, et al. Artemether ameliorates type 2 diabetic kidney disease by increasing mitochondrial pyruvate carrier content in $\mathrm{db} / \mathrm{db}$ mice. Am J Transl Res. 2019;11(3):1389-402..

34. Tang BL. Targeting the Mitochondrial Pyruvate Carrier for Neuroprotection. Brain sci. 2019;9(9):238

35. Feng J, Ma Y, Chen Z, Hu J, Yang Q, Ding G. Mitochondrial pyruvate carrier 2 mediates mitochondrial dysfunction and apoptosis in high glucosetreated podocytes. Life Sci. 2019;237:116941.

36. Kitada M, Kume S, Koya D. Role of sirtuins in kidney disease. Curr Opin Nephrol Hypertens. 2014;23(1):75-9.

37. Morigi M, Perico L, Benigni A. Sirtuins in renal health and disease. J Am Soc Nephrol. 29(7):1799-809.

38. Srivastava SP, Li J, Kitada M, Fujita H, Yamada Y, Goodwin JE, Kanasaki K, Koya D. SIRT3 deficiency leads to induction of abnormal glycolysis in diabetic kidney with fibrosis. Cell Death Dis. 2018;9(10):997.

39. Jiao X, Li Y, Zhang T, Liu M, Chi Y. Role of Sirtuin3 in high glucose-induced apoptosis in renal tubular epithelial cells. Biochem Biophys Res Commun. 2016:480(3):387-93.

40. Li A, Liu Q, Li Q, Liu B, Yang Y, Zhang N. Berberine reduces pyruvate-driven hepatic glucose production by limiting mitochondrial import of pyruvate through mitochondrial pyruvate carrier 1. EBioMedicine. 2018;34:243-55. 
41. Yu SM, Bonventre JV. Acute kidney injury and progression of diabetic kidney disease. Adv Chronic Kidney Dis. 2018;25(2):166-80

42. Hwang S, Park J, Kim J, Jang HR, Kwon GY, Huh W, Kim YG, Kim DJ, Oh HY, Lee JE. Tissue expression of tubular injury markers is associated with renal function decline in diabetic nephropathy. J Diabetes Complicat. 2017;31(12): 1704-9.

43. Mori K, Nakao K. Neutrophil gelatinase-associated lipocalin as the real-time indicator of active kidney damage. Kidney Int. 2007;71(10):967-70.

44. Dekkers CCJ, Petrykiv S, Laverman GD, Cherney DZ, Gansevoort RT, Heerspink HJL. Effects of the SGLT-2 inhibitor dapagliflozin on glomerular and tubular injury markers. Diabetes Obes Metab. 2018;20(8):1988-93.

45. Vanderperre B, Bender T, Kunji ERS, Martinou J-C. Mitochondrial pyruvate import and its effects on homeostasis. Curr Opin Cell Biol. 2015;33:35-41.

46. Wilding JP. The role of the kidneys in glucose homeostasis in type 2 diabetes: clinical implications and therapeutic significance through sodium glucose co-transporter 2 inhibitors. Metab Clin Exp. 2014;63(10):1228-37.

\section{Publisher's Note}

Springer Nature remains neutral with regard to jurisdictional claims in published maps and institutional affiliations.

Ready to submit your research? Choose BMC and benefit from:

- fast, convenient online submission

- thorough peer review by experienced researchers in your field

- rapid publication on acceptance

- support for research data, including large and complex data types

- gold Open Access which fosters wider collaboration and increased citations

- maximum visibility for your research: over $100 \mathrm{M}$ website views per year

At BMC, research is always in progress.

Learn more biomedcentral.com/submissions 